\title{
Adaptive Multilevel Neuro-Fuzzy Model Predictive Control for Drinking Water Networks
}

\author{
J.M. Grosso, C. Ocampo-Martínez, V. Puig
}

\begin{abstract}
This paper presents a constrained Model Predictive Control (MPC) strategy enriched with soft-control techniques as neural networks and fuzzy logic, to incorporate self-tuning capabilities and reliability aspects for drinking water transport network management. The system is a multilevel controller with three hierarchical layers: neural level, fuzzy level, MPC level. Results in the Barcelona Water Network have shown that the quasi-explicit nature of the proposed predictive controller leads to improve the computational time, especially when the complexity of the problem structure can vary while tuning the receding horizons.
\end{abstract}

\section{INTRODUCTION}

Drinking Water Networks (DWNs) are large-scale multisource/multi-node flow systems which must be reliable and resilient while being subjected to constraints and continuously varying conditions with both deterministic and probabilistic nature [1]. Optimal management of these systems is a complex task and has become an increasingly environmental and socio-economic research subject worldwide [2]-[8], with special attention to efficient handling of energetic and natural resources in dense urban areas, such as Barcelona city.

Nowadays, due its systematic and practical formulation, Model Predictive Control (MPC) is becoming a strong approach to deal with challenging multi-criteria problems of real chain supply dynamic systems [9]-[17]. Nevertheless, the ever growing complexity of mathematical models (dimensionality, information structure constraints, uncertainty), turns these kind of problems costly to solve in practice, especially when controlling large-scale interconnected systems seeking the best operational policies as in transport water networks, where current MPC algorithms are not prepared enough to face the important computational burden when design aspects (i.e., set-points, prediction horizons, weights, and system topology size) have to be continually redefined.

These design issues are mitigated in this work by using soft computing techniques (i.e., neural networks and fuzzy logic). Here, MPC controllers are improved by using a priori knowledge and by exploiting the structural properties of the plant to achieve a flexible and reliable controller as a decision-support tool for the management of the DWN of Barcelona as case study.

This work has been supported by Spanish research project WATMAN (CICYT DPI2009-13744) of the Science and Technology Ministry and the DGR of Generalitat de Catalunya (SAC group Ref. 2009/SGR/1491).

J. M. Grosso, C. Ocampo-Martínez and V. Puig are with the Advanced Control Systems Research Group (SAC), at the Institut de Robòtica i Informàtica Industrial (CSIC-UPC). Llorens i Artigas 4-6, 08028. Barcelona, Spain. (e-mail:jgrosso@iri.upc.edu).
The main contribution of this paper is a Multilevel NeuroFuzzy MPC (NF-MPC) with auto-tuning capabilities to assure service reliability of the network while optimizing resources, and with less computational burden on-line due its quasi-explicit nature. The proposed architecture includes modules for demand forecasting and safety tank volumes on-line computation. The desired control specifications are expressed through performance indices associated to management policies such as assurance of water supply and reductions in control and economic costs.

\section{DWN CONTROL PROBLEM}

\section{A. DWN Linear Discrete-Time Model}

The control-oriented model of a water transport system is a simplified but representative model of the non-linear dynamic behavior, which allows predicting the effect of control actions on the entire network. Modeling principles of DWNs have been reported in the literature [18]-[19]. Considering the aforementioned references, the model of a DWN in discrete-time state space may be written as

$$
\begin{gathered}
\mathbf{x}_{k+1}=\mathbf{A} \mathbf{x}_{k}+\mathbf{B} \mathbf{u}_{k}+\mathbf{B}_{p} \mathbf{d}_{k}, \\
\mathbf{E}_{1} \mathbf{u}_{k}+\mathbf{E}_{2} \mathbf{d}_{k}=\mathbf{0}, \\
\mathbf{x}^{\text {min }} \leq \mathbf{x}_{k} \leq \mathbf{x}^{\max }, \\
\mathbf{u}^{\text {min }} \leq \mathbf{u}_{k} \leq \mathbf{u}^{\max }
\end{gathered}
$$

where $\mathbf{x} \in \mathbb{R}^{n}$ is the measurable state vector of water stock levels in $\mathrm{m}^{3}$ corresponding to the $n$ buffer tanks at time $\mathbf{k} \in \mathbb{Z}^{+}$, subject to physical constraints $\mathbf{x}^{\min }$ and $\mathbf{x}^{\max }$; $\mathbf{u} \in \mathbb{R}^{m}$ is the vector of manipulated flows in $\mathrm{m}^{3} / \mathrm{s}$ through the $m$ actuators subject to operational constraints $\mathbf{u}^{\text {min }}$ and $\mathbf{u}^{\max } ; \mathbf{d} \in \mathbb{R}^{p}$ corresponds to the vector of the $p$ water demands (sectors of consume) in $\mathrm{m}^{3} / \mathrm{s} ; \mathbf{A}, \mathbf{B}$ and $\mathbf{B}_{p}$ are state-space system matrices of suitable dimensions; and $\mathbf{E}_{1}$ and $\mathbf{E}_{2}$ are matrices of suitable dimensions dictated by the network topology.

\section{B. DWN Operational Control}

The main goal of the operational control of water transport networks is to satisfy the demands at consumer sectors, but optimizing at the same time, management policies expressed as a multi-objective control problem. Hence, MPC is a suitable technique to control a DWN because its capability to deal efficiently with multivariable dynamic constrained systems and predict the proper actions to achieve the optimal performance according to a user defined cost function. 
Specifically, the interest is to minimize the following objectives [7]:

$$
\min _{\Delta \mathbf{u}, \boldsymbol{\varepsilon}}\left[\sum_{i=0}^{H_{u}-1} f_{1}(k+i)+\sum_{i=0}^{H_{p}} f_{2}(k+i)+\sum_{i=0}^{H_{u}-1} f_{3}(k+i)\right]_{(2)}
$$

where $H_{p}$ and $H_{u}$ are the prediction and control horizons, respectively; index $k$ represents the current time instant while index $i$ represents the predicted time along the horizons; $f_{1}(k)=\left\|\left(\boldsymbol{\alpha}_{1}+\boldsymbol{\alpha}_{2}(k)\right) \mathbf{u}(k) \Delta t\right\|_{\mathbf{W}_{e}}^{1}$ minimizes the economic cost of network operation taking into account water production costs $\left(\boldsymbol{\alpha}_{1}\right)$ and water pumping electric costs $\left(\boldsymbol{\alpha}_{2}\right) ; f_{2}(k)=\|\varepsilon(k)\|_{\mathbf{W}_{x}}^{2}$ is a performance index which penalizes the amount of volume $\varepsilon$ that goes down from a safety volume value; $f_{3}(k)=\|\Delta \mathbf{u}(k)\|_{\mathbf{W}_{u}}^{2}$ minimizes control signal variations to extend actuators life and assure a smooth operation; $\mathbf{W}_{e}, \mathbf{W}_{x}$, and $\mathbf{W}_{u}$ are diagonal weighting matrices included to prioritize the objectives.

With the above information, the MPC design follows a systematic procedure [20]-[21], which generates the control input signals to the plant by combining a prediction model and a receding-horizon control strategy. The cost function (2) subject to (1) represents the desired system performance over a future horizon. Once the minimization is performed, only the first computed control action is implemented and the system operates with this constant input until the next sampling instant. Then, the optimization is solved again with new feedback measurements to compensate for unmeasured disturbances and model inaccuracies. This scheme is repeated at each future sampling period.

Receding horizon control strategy is a very easy and intuitive formulation, but on-line tuning of an MPC controller is not trivial or systematic. The MPC tuning parameters for a given cost function are usually: prediction and control horizons, set-points, and weighting matrices. The general approach is to define these parameters as constants for all the system operation but this could lead to an increase of the suboptimality due to maneuverability reduction. Other common method is to generate the complete Pareto front and select the best solution according to an extra criterion, but this method is computationally prohibited in fast dynamic or large-scale systems as the one considered in this paper. Therefore, next sections present a self-tunable MPC controller enhanced with soft computing techniques such as neural networks and fuzzy logic.

\section{Safety Volumes and Service Reliability}

There is the need to guarantee a safety water stock in each tank of the network in order to decrease the probability of shortages (when a tank or a node has not sufficient water to satisfy external demands or the transfer request coming from other tank/node) due uncertain events. To determine the amount of safety water stocks, an inventory planning strategy is addressed here to enrich previous control approaches [4], [7], [23], with replenishment policies [24]. The goal is to dynamically allocate the minimal volume in each storage unit to avoid stockouts.
An economic optimization layer with short-term prediction is implemented to virtually decouple each tank with three main purposes: (i) to estimate tanks net outflows, (ii) to compute ideal safety stocks based on demand forecasting error deviation and a given service level, and (iii) to set each base-stock introduced in the MPC problem as a soft constraint. The last purpose lets the system employ safety volumes on demand to face uncertainties. The aforesaid is expressed in the following equations:

$$
\begin{gathered}
\mathbf{x}_{s}(k)=\mathbf{D}_{L}(k)+\mathbf{S S}(k), \\
\mathbf{S S}(k)=F_{s}^{-1}(C S L) \boldsymbol{\sigma}(k), \\
\boldsymbol{\sigma}(k)=\sqrt{\boldsymbol{\sigma}_{D}^{2}(k) \mathbf{L}(k)+\boldsymbol{\sigma}_{L}^{2}(k) \mathbf{D}_{L}(k),}
\end{gathered}
$$

and

$$
\mathbf{x}(k) \geq \mathbf{x}_{s}(k)-\boldsymbol{\varepsilon}(k) \geq \mathbf{0},
$$

where $\mathbf{x}_{s} \in \mathbb{R}^{n}$ is the vector of base-stocks in $\mathrm{m}^{3}, \mathbf{D}_{L} \in \mathbb{R}^{n}$ is the vector of net lead-time forecasted demands in $\mathrm{m}^{3}$; SS $\in \mathbb{R}^{n}$ is the vector of safety stocks in $\mathrm{m}^{3} ; \boldsymbol{\sigma} \in \mathbb{R}^{n}$ is the forecast error standard deviation; $\mathbf{L} \in \mathbb{R}^{n}$ is the vector of lead-times; $\boldsymbol{\sigma}_{D}, \boldsymbol{\sigma}_{L} \in \mathbb{R}^{n}$ are the standard deviations of demands, and lead-times respectively; $F_{s}^{-1}$ is the inverse cumulative normal distribution, $C S L$ is the desired customer service level (percentage of customers that do not experience a stockout) and $\varepsilon$ is the vector of slack variables introduced to relax the state constraint $\mathbf{x}(k) \geq \mathbf{x}_{s}(k)$, i.e., $\boldsymbol{\varepsilon}$ represents the amount of volume that goes down from the desired base-stock per each tank and $\varepsilon=0$ if no violation of this constraint occurs.

\section{Auto-Tuned Multilevel Neuro-Fuzzy MPC}

In order to achieve a flexible and reliable controller as a decision-support tool for the management of DWNs, a Multilevel Neuro-Fuzzy MPC (NF-MPC) with auto-tuning capabilities and less on-line computational burden is proposed in this section. NF-MPC is a quasi-explicit controller that combines the advantages of conventional MPC and knowledge-based soft control.

The proposed controller is based on a three-level hierarchical control architecture (see Fig. 1). The first level is a Neural Level for strategic determination of variable set-points and demand forecasting, the second level consists in a Fuzzy rule-based auto-tuning of the MPC and the third level is the closed-loop control system with receding horizon approach flexible to be constrained or unconstrained.

\section{A. Neural Network for Demand Forecasting}

This module focuses on the problem of water demand forecasting for real time operation of the DWN. An hourly consumption data analysis is proposed here for training an artificial neural network multilayer perceptron (MLP) with Bayesian regulation backpropagation, which updates the weights and bias values according to Levenberg-Marquardt optimization. It minimizes a combination of squared errors and weights, and then determines the correct combination so as to produce a network that generalizes well. The inputs 


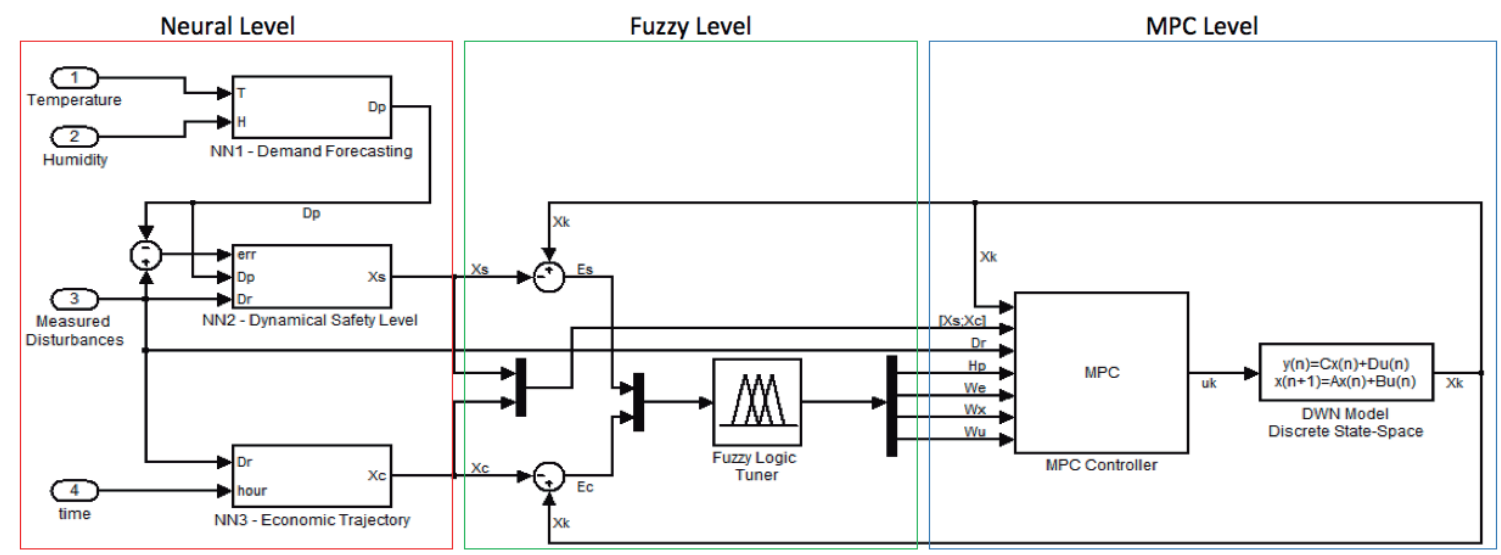

Fig. 1. Three-level hierarchical control architecture. Neuro-Fuzzy MPC

to the forecasting models are chosen based on literature review [22], and correlation analysis, considering consumption data and meteorological variables such as temperature and air relative humidity. Principal component analysis (PCA) preprocessing is applied to the training patterns.

\section{B. Neural Network for Dynamical Safety Volumes}

A Backpropagation MLP is proposed here to be trained with patterns that are generated using historical data of measured demands, forecasted demands and forecasting errors as inputs, while using safety inventories as targets calculated for a $95 \%$ service level and four hours lead-time demand by stock management methodologies [24]. The goal with this neural modeling is to avoid on-line optimizations which are required to virtually decouple the tanks for safety stock calculations in section II-C, and to use the non-linear explicit model of the trained MLP to set dynamically the safety reference volumes.

\section{Neural Network for Optimal Economic Trajectory}

The optimal economic trajectory of water volume in each tank is the one obtained considering only the first objective in the MPC problem related to a DWN solving an LP constrained optimization problem on-line. With the intention to reduce computational effort, an MLP is trained offline with the economic optimizer minimizing $f_{1}$ in (2). A nonlinear explicit model of the optimal operation of the network is obtained taking into account the hourly electric tariff and the measured demand pattern as inputs.

\section{Fuzzy rule-based Tuner for DWN-MPC Controller}

Self-tuning on-line algorithms for MPC of large-scale networked systems is not a widely reported topic in literature. Most of the tuning strategies in multi-objective optimization problems [25] take into account the exploration of the complete Pareto front to choose a non-dominated solution in line with the management objectives. The aim behind the Pareto frontier applied to MPC of a DWN is to find a direct relation between the weights of the solution points and the water demands [7].

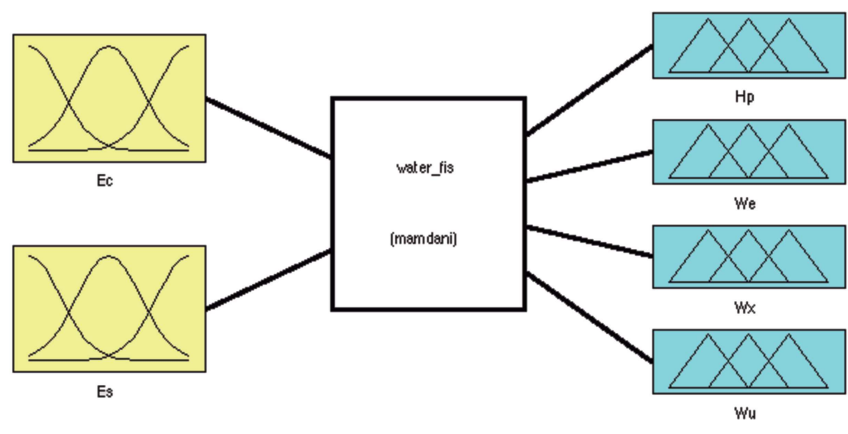

Fig. 2. Fuzzy system for automatic tuning of MPC controller

In this work, a knowledge-based soft control technique is presented. A fuzzy inference system (FIS) interacts with the MPC Level by automatically adjusting the tuning parameters of the controller based on the output feedback and the measured disturbances. For more detailed explanation on fuzzy inference reasoning, the reader could refer to [26].

1) Fuzzy System: This knowledge-based soft tuner for the MPC has been proposed after experience and understanding the effect of the tuning parameters on the performance of the controlled system. It consists of: (i) two inputs fuzzy sets $E_{c}$ and $E_{s}$ used in the fuzzification of the economic trajectory tracking error and the safety volume tracking error, respectively; (ii) four fuzzy sets $H_{p}, W_{e}, W_{x}, W_{u}$ used in the defuzzification task to obtain the quantitative value of the tuned parameters (prediction horizon, weighting matrix for the economic objective, weighting matrix for safety storage term penalization and weighting matrix for set-points stability, respectively); and (iii) a fuzzy inference system based on sixteen logic rules.

2) Fuzzy Sets: A fuzzy set for every input and output has been designed in order to perform fuzzy inference methodology. Gaussian functions are used here for the inputs because of its smoothness while triangular and trapezoidal functions are used for the outputs. The universe of discourse for the input $E_{c}$ and output $H_{p}$ is: small $(S)$, medium $(M)$, large $(L)$; for the input $E_{s}$ is: large-negative $(L N)$, mediumnegative $(M N)$, small-negative $(S M)$, very-small-negative 
TABLE I

FUZZY-LOGIC RULES FOR NF-MPC

\begin{tabular}{lll}
\hline 1 & $\left\{E_{c}=L, E_{s}=L P\right\}$ & $\Rightarrow\left\{H_{p}=L, W_{e}=L, W_{x}=V S, W_{u}=V S\right\}$ \\
2 & $\left\{E_{c}=L, E_{s}=M P\right\}$ & $\Rightarrow\left\{H_{p}=L, W_{e}=L, W_{x}=V S, W_{u}=S\right\}$ \\
3 & $\left\{E_{c}=L, E_{s}=S P\right\}$ & $\Rightarrow\left\{H_{p}=L, W_{e}=M, W_{x}=V S, W_{u}=M\right\}$ \\
4 & $\left\{E_{c}=L, E_{s}=V S P\right\}$ & $\Rightarrow\left\{H_{p}=L, W_{e}=S, W_{x}=S, W_{u}=L\right\}$ \\
5 & $\left\{E_{c}=M, E_{s}=L P\right\}$ & $\Rightarrow\left\{H_{p}=M, W_{e}=L, W_{x}=V S, W_{u}=V S\right\}$ \\
$6\left\{E_{c}=M, E_{s}=M P\right\}$ & $\Rightarrow\left\{H_{p}=M, W_{e}=L, W_{x}=V S, W_{u}=S\right\}$ \\
7 & $\left\{E_{c}=M, E_{s}=S P\right\}$ & $\Rightarrow\left\{H_{p}=M, W_{e}=M, W_{x}=V S, W_{u}=M\right\}$ \\
$8\left\{E_{c}=M, E_{s}=V S P\right\}$ & $\Rightarrow\left\{H_{p}=M, W_{e}=S, W_{x}=S, W_{u}=L\right\}$ \\
$9\left\{E_{c}=S, E_{s}=L P\right\}$ & $\Rightarrow\left\{H_{p}=S, W_{e}=L, W_{x}=V S, W_{u}=V S\right\}$ \\
$10\left\{E_{c}=S, E_{s}=M P\right\}$ & $\Rightarrow\left\{H_{p}=S, W_{e}=M, W_{x}=V S, W_{u}=S\right\}$ \\
$11\left\{E_{c}=S, E_{s}=S P\right\}$ & $\Rightarrow\left\{H_{p}=S, W_{e}=M, W_{x}=V S, W_{u}=M\right\}$ \\
$12\left\{E_{c}=S, E_{s}=V S P\right\}$ & $\Rightarrow\left\{H_{p}=S, W_{e}=S, W_{x}=S, W_{u}=L\right\}$ \\
$13\left\{E_{s}=L N\right\}$ & $\Rightarrow\left\{H_{p}=L, W_{e}=V S, W_{x}=L, W_{u}=V S\right\}$ \\
$14\left\{E_{s}=M N\right\}$ & $\Rightarrow\left\{H_{p}=L, W_{e}=V S, W_{x}=L, W_{u}=V S\right\}$ \\
$15\left\{E_{s}=S N\right\}$ & $\Rightarrow\left\{H_{p}=L, W_{e}=V S, W_{x}=L, W_{u}=V S\right\}$ \\
$16\left\{E_{s}=V S N\right)$ & $\Rightarrow\left\{H_{p}=L, W_{e}=V S, W_{x}=L, W_{u}=V S\right\}$ \\
\hline & &
\end{tabular}

$(V S N)$, very-small-positive $(V S P)$, small-positive $(S P)$, medium-positive $(M P)$, large-positive $(L P)$; for the outputs $W_{e}, W_{x}, W_{u}$ is: very small $(V S)$, small $(S)$, medium $(M)$, large $(L)$.

3) Fuzzy Rules: The logic statements proposed in this work are presented in Table I. These rules are setted taking into account the expert knowledge acquired after several simulation scenarios for the optimal operation of a water network.

\section{SIMULATION AND RESULTS}

In this section, simulation results are presented. The selected case study is an aggregate model of the DWN of Barcelona, which consists of 17 tanks, 61 actuators, 25 measured demands and 11 nodes (see Fig. 3). All the simulations have been done over a time period of four days (96 hours). The selected sampling time is one hour. Simulations have been carried out using the TOMLAB ${ }^{\circledR} 7.6$ optimization package, Fuzzy Logic Toolbox ${ }^{\mathrm{TM}}$ and Neural Network Toolbox ${ }^{\text {TM }}$ for Matlab ${ }^{\circledR}$ R2010b (64 bits). The computer used to run the simulations is a PC Intel ${ }^{\circledR}$ Core $^{\mathrm{TM}}$ E8600 running both cores at $3.33 \mathrm{GHz}$ with $8 \mathrm{~GB}$ of RAM.

\section{A. Demand Forecasting}

Forecasted water demand is calculated based on meteorological (temperature and air relative humidity) historical data and predictions available from the Servei de Meteorologia de Catalunya (www.meteocat.com). Figure 4 shows that the prediction is nervous in some points but the magnitud of the forecasting error is not a reason to reject the obtained model. In fact, these minimal discrepancies are reflected in an increase of the safety volume.

\section{B. Economic Trajectory and Safety Volumes Modeling}

Neural modeling schemes for the economic optimal trajectory and the safety volume trajectory performs with high accuracy as it could be expected (see Fig. 5), because both MLPs are trained using optimizers or explicit models instead of experimental driven data as in the forecasting demand case. The advantage of using these neural models is that

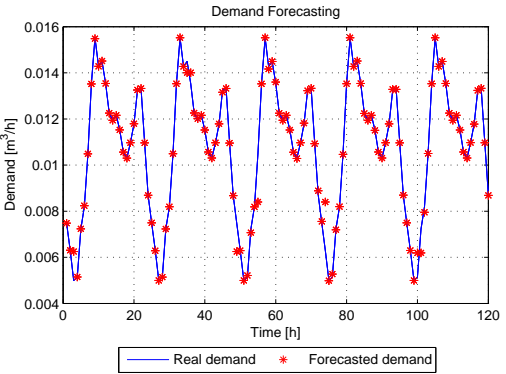

Fig. 4. Demand forecasting for c125PAL consumer sector. Real (blue), Forecasted (red)

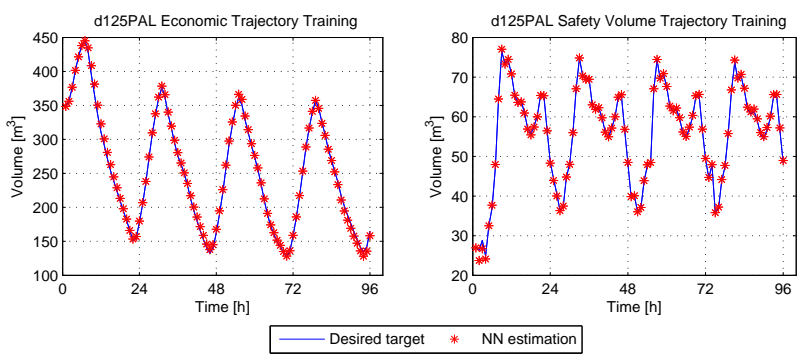

Fig. 5. Neural Network results for economic trajectory and safety volumes

the time invested in processing data and training the neural system will be gained in the on-line solving process once the neural networks are accurately validated and tested.

\section{Auto-Tuning Results}

The parameters tuned in the MPC problem are $H_{p}, W_{e}, W_{x}, W_{u}$, whose histograms are shown in Fig.6. Results for the NF-MPC with an unmeasured random disturbance of at most $20 \%$ of the demand pattern are obtained.

In most of the results presented in literature for MPC, tuning is focused on the weighting matrices and most of the times with no adaptation schemes. Nevertheless, for large-scale systems, an efficient selection of the horizons is also demanded because the size and complexity of the optimization problem is based mainly in this parameter.

\section{NF-MPC Controller for DWN}

The proposed Neuro-Fuzzy approach has been implemented for the tuning of a constrained MPC to operate the aggregate model of the Barcelona DWN. Results have been compared with two previous strategies. The controllers are the following ones:

- MPCo: original approach of MPC with fixed prediction and control horizons $(24 \mathrm{~h})$, constant safety water stocks and constant weights for the prioritization of management objectives.

- MPCss: a Two-level MPC which implements analytically the dynamic optimization of safety stocks following II-C. It considers fixed horizons (24h) and weights.

- NF-MPC: Neuro-Fuzzy MPC with self-tuning capabilities to adjust the parameters stated in III. 


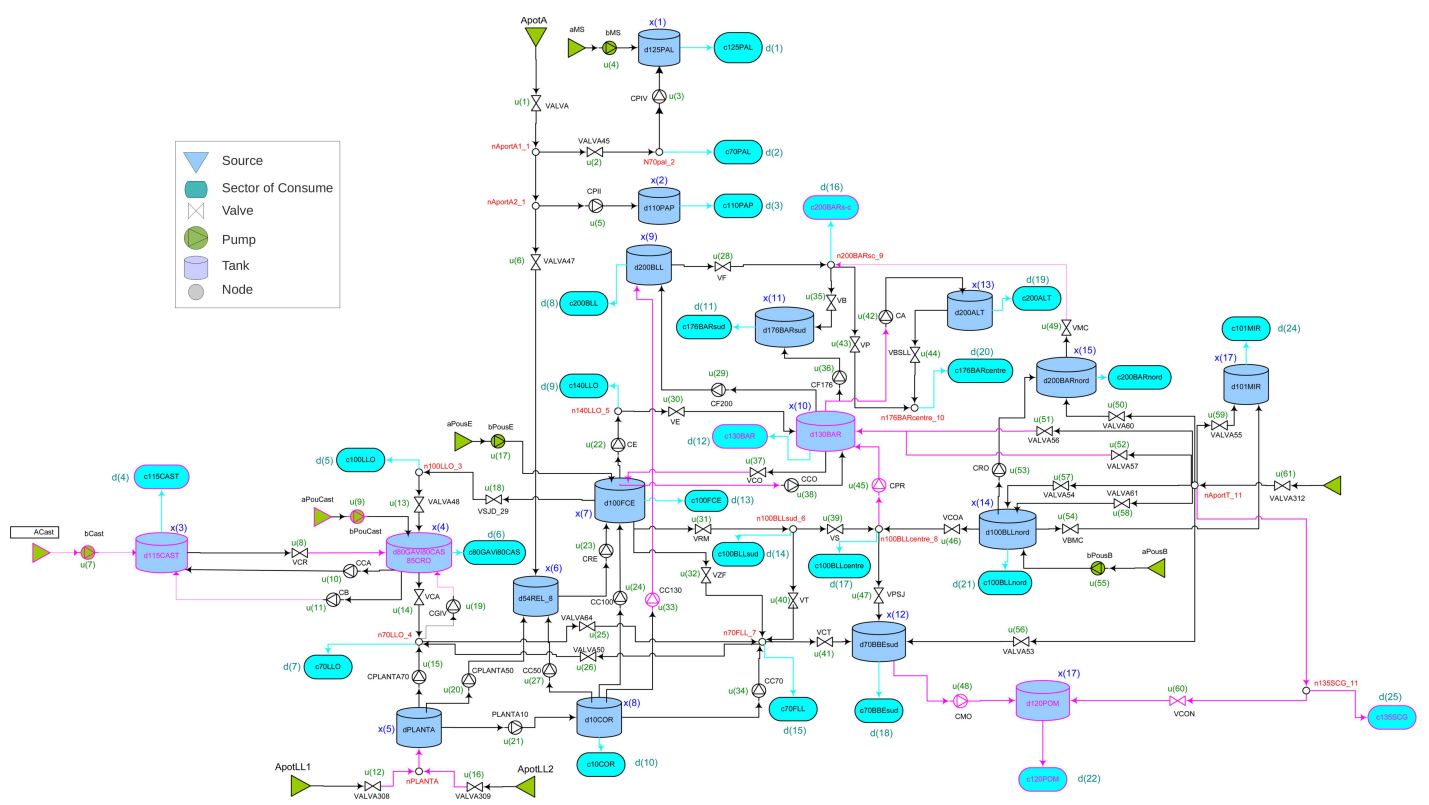

Fig. 3. Case Study: Aggregate model of the DWN of Barcelona
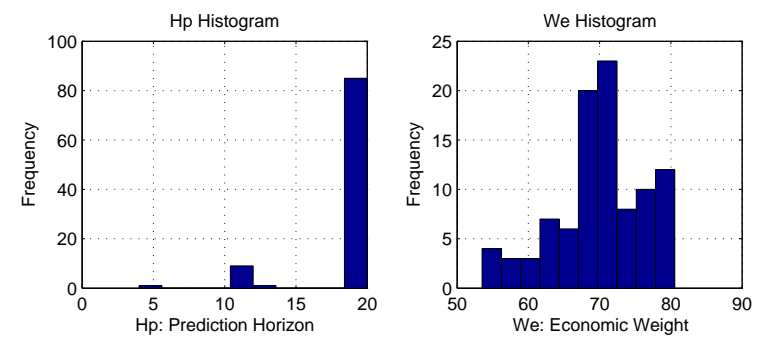

Wx Histogram
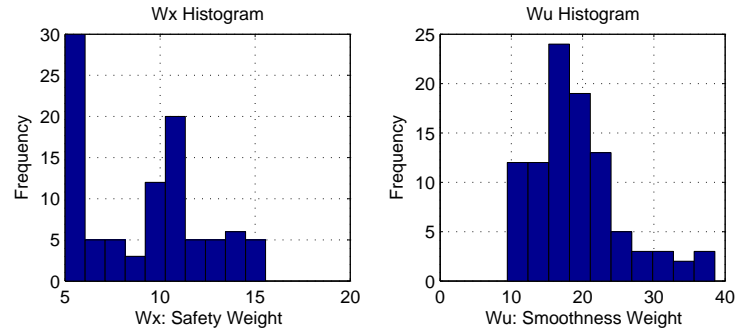

Fig. 6. Histograms of tuning parameters for the NF-MPC

Table II shows the specific key performance indicators for the aforementioned controllers. Simulations show that fixed parameters such as safety stocks, weights and horizons are a drawback for the management of complex systems. Figure 7 shows the control strategies for the operation of two tanks. Figure 8 presents the operation of two actuators, showing the effectiveness of the strategy to decide pumping actions in periods where electric tariff is lower.

The MPCo controller presents the highest economic cost due to the static safety volumes strategy that limit the solution space to achieve economic optimization. This approach do not guarantee optimal results for any condition because the safety is fixed heuristically without taking into account demand variations.
TABLE II

KEY PERFORMANCE INDICATORS FOR THE DIFFERENT APPROACHES

\begin{tabular}{ccccc}
\hline Controller & $\begin{array}{c}\text { Economic }\left(10^{3}\right) \\
\text { [e.u.] }\end{array}$ & Safety & Smoothness & $\begin{array}{c}\text { Time } \\
{[\mathrm{s}]}\end{array}$ \\
\hline MPCo & 183.74 & 28.8022 & 0.1318 & 142.01 \\
MPCss & 176.77 & 5.0295 & 0.1340 & 286.17 \\
NF-MPC & 178.99 & 5.2138 & 0.1172 & 132.91 \\
\hline
\end{tabular}
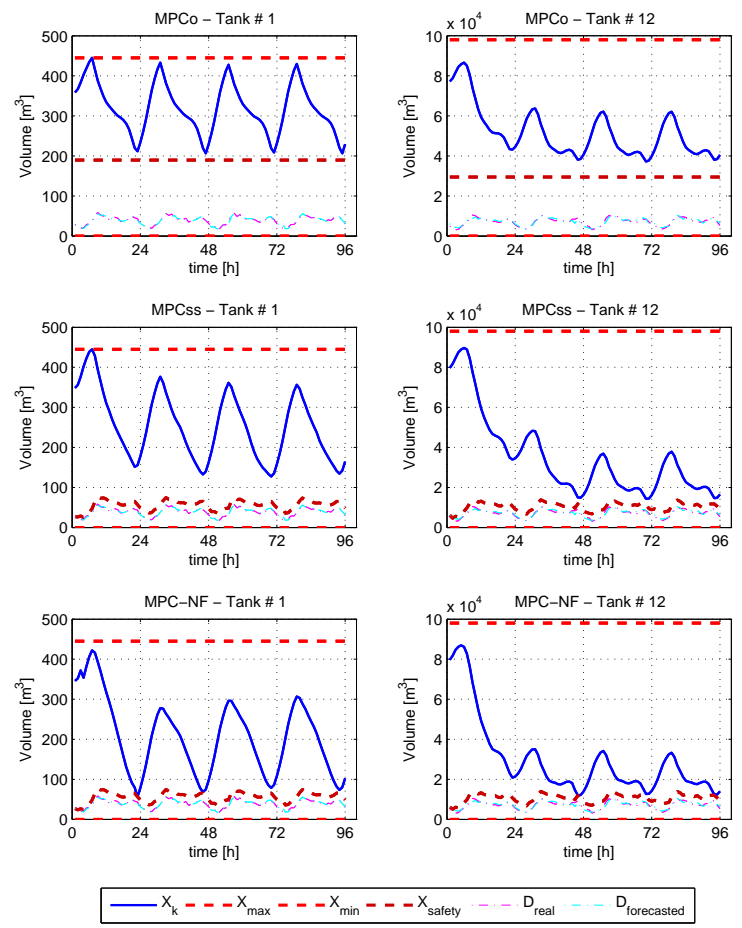

Fig. 7. Dynamic variation of tanks volumes for the different approaches 


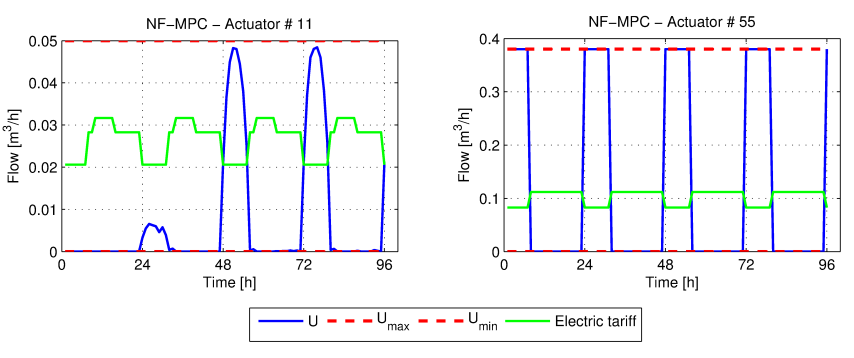

Fig. 8. Control actions for the NF-MPC with adaptive parameters

The MPCss controller is robust to disturbance uncertainty and presents the best economic performance but the highest computational effort since it involves more on-line optimization problems to set safety stocks.

The NF-MPC controller outperforms the previous strategies. It presents similar results to the MPCss for the economic, safety and smoothness indicators but reduces the computational burden. NF-MPC does not require explicit formulation of constraints in an optimizer routine and it is flexible to self-adapt controller parameters if the operational conditions change. This capability helps managers to deal with demand uncertainty and prediction errors in an optimal and economic way.

\section{CONCLUDING REMARKS}

This paper has presented a Multilevel Neuro-Fuzzy Model Predictive Controller with self-tuning capabilities for the efficient management of water transport systems. The resultant controller architecture has been applied to an aggregate model of the Barcelona DWN obtaining important improvements in the computation time towards on-line implementation for large scale systems. The selected parameters to be tuned in the MPC problem were the prediction horizon and the weighting matrices of the multiojective cost function. The main advantage of the fuzzy tuner is that it is able to tune every element independently, which is a difficult task in analytical approaches due their lack of intuitiveness for multivariable large-scale systems. The proposed scheme is a quasi-explicit MPC because most of the heavy computational tasks are converted into non-linear explicit modules using neural networks. The controller also tunes the setpoints based on inventory management theory, enriching the controller design with reliability aspects to assure a customer service level under disturbances uncertainty. Further research will be done in reinforcement learning to adapt and to improve the fuzzy rules-based level.

\section{REFERENCES}

[1] J. van Zyl, O. Piller, Y. le Gat. "Sizing Municipal Storage Tanks Based on Reliability Criteria". Journal of Water Resources Planning and Management, Vol. 134, No. 6., 2008.

[2] C. Ocampo-Martínez, D. Barcelli, V. Puig and A. Bemporad. "Hierarchical and decentralised model predictive control of drinking water networks: application to Barcelona case study". IET Control Theory and Applications, 6(1): 62-71. 2012.

[3] C. Ocampo-Martínez, S. Bovo, and V. Puig. "Partitioning approach oriented to the decentralised predictive control of large-scale systems". Journal of Process Control, 21(5): 775-786. 2011.
[4] J. Pascual, J. Romera, V. Puig, R. Creus, M. Minoves. "Operational Predictive Optimal Control of Barcelona Water Transport Network". Proc. 18th IFAC World Congress, Milan, Italy. 2011.

[5] J. Vieira, M. Cunha, L. Nunes, J. Monteiro, L. Ribeiro, T. Stigter, J. Nascimento, and H. Lucas. "Optimization of the Operation of Large-Scale Multisource Water-Supply Systems". Journal of Water Resources Planning and Management. 137, 150, 2011.

[6] G. Cembrano, J. Quevedo, V. Puig, R. Pérez, J. Figueras, J.M. Verdejo i Rabassó, M.I. Escaler, G. Ramón, G. Barnet, P. Rodríguez and M. Casas. "PLIO: a generic tool for real-time operational predictive optimal control of water networks". Water Science and Technology, 64(2): 448-459, 2011.

[7] R. Toro, C. Ocampo-Martinez, F. Logist, J. Van Impe, V. Puig. "Tuning of Predictive Controllers for Drinking Water Networked Systems", Proc. 18th IFAC World Congress, Milan, Italy. 2011.

[8] D. Barcelli, C. Ocampo-Martínez, V.Puig and A. Bemporad. "Decentralized model predictive control of drinking water networks using an automatic subsystem decomposition approach". 12th IFAC Symposium on Large-Scale Systems: Theory and Applications, 1-6, 2010.

[9] S. Tzafestas, G. Kapsiotis, E. Kyriannakis. Model-based predictive control for generalized production planning problems. Computers in Industry 34, 201-210, 1997.

[10] E. Perea-López, B.E. Ydstie and I.E. Grossman. "A model predictive control strategy for supply chain optimization". Computers and Chemical Engineering 27, 1201-1218, 2003.

[11] P. Seferlis and N.F. Giannelos. "A two-layered optimisation-based control strategy for multi-echelon supply chain networks". Computers and Chemical Engineering 28, 799-809, 2004.

[12] E. Aggelogiannaki, P. Doganis, and H. Sarimveis. "An adaptive model predictive control configuration for production-inventory systems". International Journal of Production Economics 114, 165-178, 2008.

[13] W. Wang, D.E. Rivera and K.G. Kempf. "Model Predictive Control strategies for supply chain management in semiconductor manufacturing”. International Journal of Production Economics 107 (1), 56-77, 2007.

[14] W. Wang and D.E. Rivera. "Model Predictive Control for tactical decision-making in semiconductor manufacturing supply chain management". IEEE Transactions on Control Systems Technology 16 (5), 841-855, 2008.

[15] C. Stoica, M.R. Arahal, D.E. Rivera, P. Rodríguez-Ayerbe, and D. Dumur, "Application of Robustified Model Predictive Control to a Production-Inventory System". 48th IEEE Conference on Decision and Control and 28th Chinese Control Conference, Shanghai, P.R. China, December 16-18, 2009.

[16] J.D. Schwartz and D.E. Rivera. "A process control approach to tactical inventory management in production-inventory systems". International Journal of Production Economics 125, 111-124, 2010.

[17] Alessandri, M. Gaggero, F. Tonelli. "Min-Max and Predictive Control for the Management of Distribution in Supply Chains". IEEE Transactions on Control Systems Technology, 19, 1075 - 1089, 2011.

[18] M. Brdys, and B. Ulanicki. "Operational control of water systems: structures, algorithms and applications". Prentice Hall, 1994.

[19] Fambrini, C. Ocampo-Martínez, D. Barcelli, and V. Puig. "Model predictive control of drinking water networks: A hierarchical and decentralized approach", 2010 American Control Conference, Baltimore MD, USA, pp. 3951-3956, IEEE, 2010.

[20] E. F. Camacho and C. Bordons, "Model Predictive Control", 2nd ed. Springer-Verlag, 2004.

[21] J.M. Maciejowski, "Predictive control with constraints". Prentice Hall, 2002.

[22] M. Babel and V. Shinde. "Identifying Prominent Explanatory Variables for Water Demand Prediction Using Artificial Neural Networks: A Case Study of Bangkok". Journal of Water Resources Management, 25, 1653 - 1676, 2011.

[23] J. Adrian. "Enhancement of management criteria in the MPC design for Predictive Controllers of drinking water networks". Master's thesis, Universitat Politécnica de Catalunya, 2010.

[24] M. Christopher. "Logistics and supply chain management: creating value-adding networks". 4th ed. Pearson Education, 2011.

[25] M. Ehrgott. "Multicriteria Optimization". Second Edition. Springer Berlin. 2005.

[26] S. S. Farin Wata, R. Langari and D. P. Filev. "Fuzzy Control: Synthesis and Analysis". 1st Edition. John Wiley \& Sons, Inc. 2000. 